\title{
Dislocation Patterns and the Similitude Principle: 2.5D Mesoscale Simulations
}

\author{
D. Gómez-García, ${ }^{1}$ B. Devincre, ${ }^{2}$ and L.P. Kubin ${ }^{2}$ \\ ${ }^{1}$ Departamento de Física de la Materia Condensada-Universidad de Sevilla, Apartado 1065, 41080 Sevilla, Spain \\ ${ }^{2}$ Laboratoire d'Étude des Microstructures, CNRS-ONERA, 29 Avenue de la Division Leclerc,
}

Boîte Postale 72, 92322 Châtillon Cedex, France

(Received 6 December 2005; published 30 March 2006)

\begin{abstract}
During plastic flow of crystalline solids, dislocations self-organize in the form of patterns, with a wavelength that is inversely proportional to stress. After four decades of investigations, the origin of this property is still under discussion. We show that dislocation patterns verifying the principle of similitude can be obtained from dynamics simulations of double slip. These patterns are formed in the presence of long- and short-range interactions, but they are not significantly modified when only short-range interactions are present. This new insight into dislocation patterning phenomena has important implications regarding current models.
\end{abstract}

DOI: 10.1103/PhysRevLett.96.125503

During plastic flow of crystalline solids, dislocations, the linear defects that carry plasticity at the microscopic scale, move and multiply. In parallel, dislocation microstructures tend to self-organize in the form of patterns containing dislocation-rich and dislocation-poor regions. As elementary dislocation processes are reasonably well understood [1], this collective behavior constitutes a major obstacle to a physical modeling of the mechanical response. In monotonic deformation and in multislip conditions, the patterns consist of three-dimensional cells bounded by cell walls. A wealth of experimental observations [2] shows that during plastic deformation, the average spacing between cell walls shrinks like the inverse of the recorded stress, the flow stress. This property is known under the name of principle of similitude [3]. After many controversies about how to model the formation of dislocation cells (see Ref. [4] for a review), it was realized 20 years ago that dislocation patterning is an example of self-organization in a system driven far from equilibrium. In the models that were further developed, the main difficulty consists in accounting for long-range interaction stresses between dislocations, which were assumed to be at the origin of patterning phenomena $[5,6]$. The specific features of such models are, however, still under discussion, as they do not account for a few essential properties of dislocations.

Dislocation dynamics simulations constitute a natural tool for investigating this type of collective behavior. However, 3D simulations are still too demanding in terms of computational load to allow investigating the similitude principle. A study of 3D patterns in face-centered cubic (fcc) crystals has, nevertheless, confirmed that three main mechanisms, which are currently implemented in these simulations, participate to pattern formation [7]. The reactions between intersecting dislocations, or junctions, are strong obstacles which pin the cell walls. Elastic interactions between dislocations, which are long-ranged, also necessarily play a role. The cross-slip of screw dislocations stabilizes dislocation tangles, accounts for the three-
PACS numbers: 61.72.Lk, 62.20.Fe, 82.20.Wt, 89.75.Da

dimensional dissemination of slip, and governs the selforganization kinetics.

Dislocation patterns can be more easily obtained in 2D, but in drastically simplified conditions [5]. As a consequence, none of the existing 2D studies lead to patterns that consistently verify the scaling relations commonly found in deformed fcc metals. In what follows we propose a " $2.5 \mathrm{D}$ " simulation, which attempts to reproduce, as closely as possible, the relevant 3D dislocation mechanisms. Dislocation cells are obtained in conditions of double slip over a range of dislocation densities that extends over 2 orders of magnitude. It is shown that the microstructures follow the principle of similitude, which allows discussion of the main properties of the obtained patterns.

The similitude principle is written in the form

$$
\frac{\tau}{\mu}=K \frac{b}{\lambda}
$$

where $\tau$ is the flow stress, $\mu$ is the shear modulus, $b$ the modulus of the dislocations Burgers vector, and $\lambda$ the average wavelength of the pattern. The constant $K$ somehow appears as a universal constant $(K \approx 10)$. A second scaling law, the Taylor relation, which expresses the flow stress in terms of the square root of the dislocation density, $\rho$, also characterizes plastic flow

$$
\frac{\tau}{\mu}=\alpha b \sqrt{\rho}
$$

In this equation, $\alpha$ is an approximately constant coefficient. Here, $\rho$ is taken as the total density, that is, twice the density of intersecting obstacles seen by each slip system during duplex slip. In such conditions, $\alpha \approx 0.25$. The Taylor relation has been the subject of numerous experimental checks. It is well reproduced by $3 \mathrm{D}$ mesoscale simulations (see Ref. [8]) and is understood as reflecting essentially the strength of the junctions formed in increasing numbers as plastic flow proceeds. 
Parts of the present simulation are rather conventional. The simulated area is a square of side $L$ defining the two crystallographic axes [10] and [01]. Two sets of infinite edge dislocations, with slip directions $1 / 2[\sqrt{3} 1]$ and $1 / 2[-\sqrt{3} 1]$ are introduced endon in two different slip systems which have same Schmid factor $(F=\sqrt{3} / 4)$. The free-flight velocity of the dislocations, $v$, under an effective resolved stress $\tau^{*}$ is given by $\tau^{*} b=B v$, where $B$ is a phonon drag constant. The two slip systems are loaded in a symmetrical manner by applying a constant plastic strain rate along the [01] direction, which produces a total plastic strain $\varepsilon$. The effective stress on each dislocation, $\tau^{*}=\tau_{\text {app }}+\tau_{\text {int }}$, is calculated as the sum of the applied stress and the long-range elastic interaction stresses with other dislocations. Use is made of periodic boundary conditions and interaction stresses are computed with the help of the fast multipole method [9]. A cutoff radius $R_{c}$ is applied to the dislocation fields, with a value that is randomly redefined at each time step and for each segment in the range $L / 2-L / 3$. This procedure allows avoidance of the formation of artificial patterns arising from the introduction of a constant cutoff distance. For each initial dislocation density, $L$ is chosen by trial and error in such a way as to obtain a pattern of wavelength $\lambda=L / 5-L / 6$ smaller than $R_{c}$. Finally, dislocation dipoles are assumed to mutually annihilate when their height is smaller than $5 b$ [10].

At this point, what is modeled is a "dislocation gas" that does not include the main mechanisms driving pattern formation. For this reason, results from 3D dislocation dynamics simulations (see Ref. [7] for references) are used to parametrize additional local rules, specifically, the calculation of the effective stress on moving dislocations, their multiplication rate and the conditions for junction formation and destruction.

Early estimates [11], as well as 3D simulations [7], indicate that long-range interaction stresses, that is, at distances larger than the mean distance between dislocations, $\rho^{-1 / 2}$, typically contribute to only one-fifth of the resistive stress opposing dislocation motion. The major resistive contribution arises from the line tension on segments that bow out between intersecting dislocations obstacles. Hence, one has to take line tension into account in 2D. A calculation of the sum of applied and interaction stresses in 3D and 2D shows that, on average, the effective stress must be scaled down by a factor of about five in the latter in order to appropriately incorporate line tension effects.

It is generally observed, and checked by 3D simulations, that dislocation multiplication is characterized by a linear increase of the total density $\rho$ as a function of strain $d \rho / d \varepsilon=m$, where the constant $m$ is of the order of $10^{15} \mathrm{~m}^{-2}$. The value adopted here, $m=2 \times 10^{15} \mathrm{~m}^{-2}$, is taken from 3D simulations of duplex slip. Reproducing this linear evolution in 2D requires some care. For instance, introducing fixed dislocation sources amounts to making a very strong simplification. Indeed, if one considers a thin slice of 3D crystal, most of the dislocations emerging into it originate from dislocation sources located in the surrounding volume.

In 2D, each pointlike dislocation moving by $\Delta x$ during a simulation time step produces a shear increment $\Delta \gamma=$ $b \delta x / L^{2}$. The resulting total strain, $\Delta \varepsilon=F \sum \Delta \gamma$, is the projection of the total shear increment along the loading axis. During a strain increment, $\Delta n$ new dislocations appear and the instantaneous dislocation density is taken as the number of pointlike defects per unit area, $\rho=n / L^{2}$. It is justified to take the same multiplication rate both in $2 \mathrm{D}$ and 3D, because $m$ is proportional to the inverse of a length squared in the two cases. Then, $\Delta n=m L^{2} \Delta \varepsilon$. Fresh dislocations are randomly introduced in the simulated area, provided that their effective stress is in the direction of the applied shear stress. In order to satisfy mechanical equilibrium, new dislocations of each sign (as well as the initial ones), are equally distributed between the slip systems. This multiplication rule disseminates fresh mobile dislocations into the simulated area, thus mimicking the effect of the 3D dislocation flux.

Two local rules are introduced to deal with the formation and destruction of junctions. The formation criterion ensures that the probability for short-range reactions in $2 \mathrm{D}$ is of same order as in 3D. The average length of junctions under stress, as measured by 3D simulations, is $\ell_{j} \approx$ $0.2 \rho^{-1 / 2}$ [12]. In a $2 \mathrm{D}$ projection, a junction, stable or not, should connect two pointlike defects separated by a distance somehow smaller than this value. For the sake of simplicity, we assume that two attractive defects moving in different glide planes may form a junction and are blocked at their current position when their distance is $d_{j} \leq \ell_{j}$. Once a junction is formed, the interaction stress between the two dislocations is set to zero, since it is not relevant to the $3 \mathrm{D}$ process.

The second criterion deals with junction destruction $[13,14]$. A junction is effectively formed and remains stable only when the stress on each dislocation is lower than a critical value $\tau_{j}$. In $3 \mathrm{D}$, this quantity scales like the inverse of the local length $\rho_{l}^{-1 / 2}$ of the interacting segments, where $\rho_{l}$ is a local dislocation density. The local stress is then defined from a line tension relation of the form

$$
\tau_{j}=\beta \frac{\mu b}{\rho_{l}^{-1 / 2}} \ln \left(\frac{\rho_{l}^{-1 / 2}}{b}\right)
$$

where $\beta$ is a constant. The local dislocation density is obtained by defining a disk of radius $R_{j}$ centered at the junction and containing $n=12$ pointlike defects. To recover a reasonable value of the flow stress, we take $\beta=$ 0.035 . Thus, the criterion for junction description accounts for the local spatial nonuniformities in dislocation density.

Copper is taken as reference for defining the material constants $b, \mu$, and $B$. Simulations are carried out for 
different equivalent initial dislocation densities $\rho$ ranging from $5 \times 10^{11} \mathrm{~m}^{-2}$ to $5 \times 10^{13} \mathrm{~m}^{-2}$.

Figure 1(a) shows the dependence of the resolved flow stress on the square root of the total dislocation density as yielded by the numerical tests. The Taylor relation [Eq. (2)] is recovered, as expected, with a constant slope: $\alpha \approx 0.65$. This value could be fitted exactly by modifying the constant $\beta$, but an $\alpha$ value slightly larger than the experimental one was chosen in order to reduce as much as possible the wavelength of the dislocation patterns and improve the statistical quality of the results.

The total dislocation density is plotted as a function of the total plastic strain in Fig. 1(b). The small initial densities correspond to large simulated areas and are more demanding in terms of computing power, hence the small values of the maximum strains. For small densities, the curves present a constant initial slope, close to that of the implemented multiplication rate, followed by a pseudoparabolic stage, which appears earlier with increasing initial density. This stage is due to the increased number of annihilations at large densities.

Because of the use of periodic boundary conditions, the simulated dislocation microstructures self-organize when the wavelength of the pattern is commensurate with the dimension of the simulated area in the corresponding direction. With increasing dislocation density and flow stress, a pattern is progressively formed [15], of which an example is shown in Fig. 2. With increasing stress, this ordered microstructure is destroyed. Other commensurate structures are presumably formed again at larger strains, which cannot be reached by the present simulations. As shown below in Fig. 3, once a pattern is formed, its periodicity is only dictated by the principle of similitude.

The two sets of dislocation walls shown in Fig. 2(a) are not exactly parallel to the prescribed slip directions. They also have slightly different periodicities, as can be seen from the Fourier transform shown in inset. This tendency is general and the obtained patterns always exhibit some
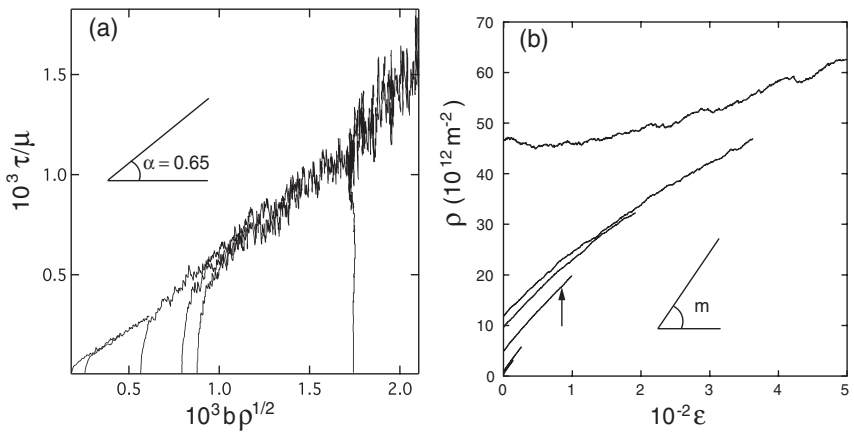

FIG. 1. (a) Shear stress vs the square root of the total dislocation density in dimensionless variables. Simulations starting with different initial densities are superimposed, yielding a linear slope $\alpha \approx 0.65$. (b) Total density vs plastic strain curves for various initial densities. The arrow indicates the conditions at which the pattern shown in Fig. 2 is obtained and $m$ is the implemented multiplication rate. asymmetry. The enlargement of a portion of wall shown in Fig. 2(b) shows that they are constituted of a majority of junctions, dislocations blocked near junctions and a few dipolar arrangements. The surface fraction of walls, as measured manually, is $f_{w}=0.3 \pm 0.05$ and does not depend on stress. Similar values are experimentally found in copper single crystals $\left(f_{w}=0.45\right)$, once cell walls are formed [16].

In another set of numerical experiments, a constant cutoff radius $R_{c}=L / 6$ is introduced, which removes the long-range interactions between dislocation walls, but preserves reactions and interactions inside walls. In such conditions, similar patterns are nevertheless formed, albeit with slightly less-well-defined walls. This confirms that pattern formation is mostly driven by short-range interactions between dislocations.

A check of the similitude principle [Eq. (1)] is shown in Fig. 3, in which the resolved shear stresses measured at the optimum point for pattern formation are plotted as a function of $1 / \lambda$ for all numerical experiments. A linear dependence is found, in fair agreement with the prediction of the similitude principle. The proportionality constant, $K=$ $6.80 \pm 0.5$, is of the same order as the experimentally measured one.

The consistency of these results can be checked through dimensionality arguments, taking into account that the surface fraction of walls is found to be a constant. If $t$ is the thickness of the walls, $f_{w}=t /(\lambda-t)$. This surface fraction can also be rewritten $f_{w}=n t / L$, where $n=L / \lambda$ is the number of walls of thickness $t$ and height $L$. If the total dislocation density is coalesced into $n$ walls with average dislocation density $\rho_{w}$, we have $\rho / \rho_{w}=n t / L=$ $f_{w}$. Thus,

$$
f_{w}=\frac{t}{\lambda-t}=\frac{\rho}{\rho_{w}}=\text { const. }
$$

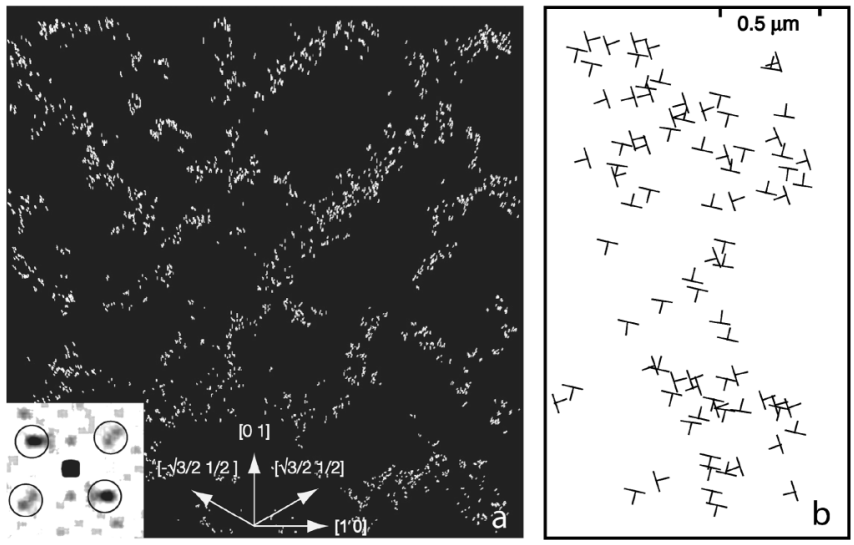

FIG. 2. (a) A periodic $2 \mathrm{D}$ pattern $(\lambda \approx 2.5 \mu \mathrm{m}, L=14 \mu \mathrm{m}$, $\rho=1.8 \times 10^{13} \mathrm{~m}^{-2}$ ). The Fourier transform of this pattern is shown in the inset; the diffraction spots corresponding to the two wall spacings are encircled. (b) Snapshot of a magnified cell wall (vertical after rotation). 


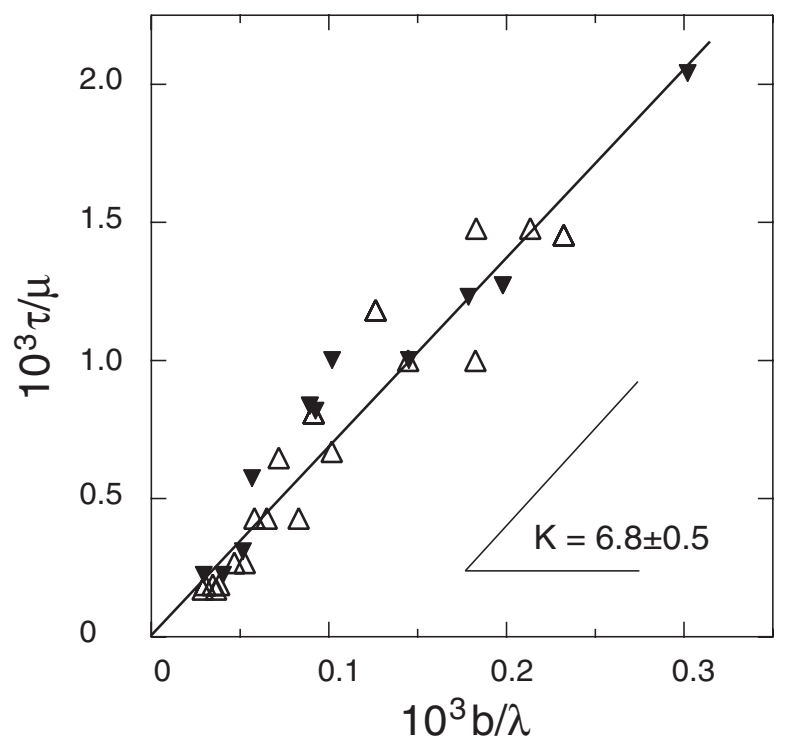

FIG. 3. Numerical check of the similitude principle: scaled resolved stress vs the inverse of the scaled wall periodicity $b / \lambda$ (two values are plotted when the two types of walls do not have exactly same periodicity). $\triangle$ : all interactions included.

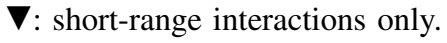

The average distance between dislocations in the walls is defined by $l_{w}=\rho_{w}^{-1 / 2}=\left(f_{w} / \rho\right)^{1 / 2}$. Then, according to Eq. (2), $l_{w} \propto 1 / \tau$. The first equality in Eq. (4) implies that the wall thickness is inversely proportional to stress if the period $\lambda$ follows the principle of similitude: $t \propto 1 / \tau$. Thus, the ratio $t / l_{w}=k$ is a constant and $l_{w}=t / k=$ $\lambda f_{w} / k\left(1+f_{w}\right)$. Combining this expression for $l_{w}$ with the one obtained above, we have $\lambda=k\left(1+f_{w}\right) / \sqrt{f_{w}} \boldsymbol{\rho}$. Finally, making use again of Eq. (2), we obtain

$$
\frac{\tau}{\mu}=\frac{\alpha k\left(1+f_{w}\right)}{\sqrt{f_{w}}} \frac{b}{\lambda} \text {. }
$$

The principle of similitude [Eq. (1)] is then recovered with $K=\alpha k\left(1+f_{w}\right) / \sqrt{f_{w}}$. With $K=6.8, \alpha=0.65$, and $f_{w}=0.3$, we obtain $k=4.4$, consistent with the values estimated from the simulated patterns [see Fig. 2(b)].

In the present work, dislocation patterning is obtained in conditions as close as possible to the three-dimensional reality. Our results strongly suggest that current models are not sufficiently specific in terms of a few relevant dislocation mechanisms. For example, only planar slip patterns can be obtained in the absence of spatial slip dissemination [17]. The incorporation of line tension effects in junction properties, relativizes the influence of dislocation dipoles and allows us to obtain reasonable numerical values for all the relevant quantities. The most important point is drawn from Fig. 3, which indicates that short-range interactions are sufficient to obtain pattern formation in multislip conditions. This aspect is absent from current models, which consider patterning as only related to long-range stresses $[5,6]$. In addition, we show that two features that are never modeled, the volume fraction of walls and the wall thickness, exhibit well-defined types of behavior. In conclusion, the present study provides both a new tool and specific guidelines for further theoretical approaches of dislocation patterning.

One of the authors (D. G.-G.) gratefully acknowledges the support of the Fundación Ramón Areces during his stay at the Laboratoire d'Étude des Microstructures.

[1] J.P. Hirth, Theory of Dislocations (Wiley, New York, 1992).

[2] S. Raj and G. Pharr, Mater. Sci. Eng. 81, 217 (1986).

[3] D. Kuhlmann-Wilsdorf, Metall. Trans. A 16A, 2091 (1985).

[4] L. Kubin, in Plastic Deformation and Fracture of Materials, edited by $\mathrm{H}$. Mughrabi ( $\mathrm{VCH}$, Weinheim (FRG), 1993), pp. 137-190.

[5] I. Groma and B. Bakó, Phys. Rev. Lett. 84, 1487 (2000).

[6] M. Zaiser and M.-Carmen Miguel, and I. Groma, Phys. Rev. B 64, 224102 (2001).

[7] R. Madec, B. Devincre, and L. Kubin, Scr. Mater. 47, 689 (2002).

[8] R. Madec, B. Devincre, and L. P. Kubin, Phys. Rev. Lett. 89, 255508 (2002).

[9] R. LeSar and J. M. Rickman, Phys. Rev. B 65, 144110 (2002).

[10] U. Essmann and H. Mughrabi, Philos. Mag. A 40, 731 (1979).

[11] G. Saada, Acta Metall. 8, 841 (1960).

[12] B. Devincre, L. Kubin, and T. Hoc, Scr. Mater. 54, 741 (2006).

[13] V. V. Bulatov, F. F. Abraham, L. P. Kubin, B. Devincre, and S. Yip, Nature (London) 391, 669 (1998).

[14] L. K. Wickham, K. W. Schwarz, and J. S. Stölken, Phys. Rev. Lett. 83, 4574 (1999).

[15] See EPAPS Document No. E-PRLTAO-96-061613 for a short movie illustrating the formation of a dislocation pattern as reproduced by the $2.5 \mathrm{D}$ simulation.

[16] R. Neuhaus and C. Schwink, Philos. Mag. A 65, 1463 (1992).

[17] A. Benzerga, Y. Bréchet, A. Needleman, and E. Van der Giessen, Model. Simul. Mater. Sci. Eng. 12, 159 (2004). 